\title{
Evaluation of Pain Following the Use of Scalpel versus Diathermy for Skin Incision in Ear, Nose, Throat and Head and Neck Surgeries
}

Diva Shrestha ${ }^{1}$

${ }^{1}$ Department of ENT and Head and Neck Surgery, Kathmandu Medical College Teaching Hospital, Sinamangal, Kathmandu, Nepal.

\section{ABSTRACT}

Background: Many studies have shown the benefits of diathermy over scalpel for making skin incisions in terms
of post operative pain and post operative analgesics requirement. The objective of the study is to compare the pain
following incision by scalpel and diathermy for skin in ENT surgery.
Methods: We conducted a prospective, randomized study and compared early post operative pain and analgesics
requirement in patients undergoing ENT and Head and Neck surgery in Department of ENT at Kathmandu Medical
College from September 2016 to August 2017 . The statistical analysis was done using MS Excel and SPSS software.
Results: Out of 65 participants, 31 were allocated in scalpel group and 30 were allocated in diathermy group. The
mean VAS score was significantly greater in scalpel group as compared to diathermy group in post operative 12,24
and 48 hours (P<0.05). The mean ketorolac requirement was significantly more in scalpel group than in diathermy
group in post operative 24 hours.
Conclusions: The early post operative pain is less in ENT-Head and Neck surgery patients with skin incision by
diathermy as compared to the patients with skin incision by scalpel.
Keywords: Diathermy; pain; post operative; scalpel.

\section{INTRODUCTION}

The surgical procedures are amenable to cause post operative pain. The inadequately relieved post operative pain can lead to various complications in the post operative period. The severity of postoperative pain is influenced by factors such as anaesthesia, analgesia and techniques of surgery including the method of incision of tissues.

Diathermy has been accepted by surgical world for bloodless cutting and removal of diseased tissues. ${ }^{1}$ Electrosurgery or diathermy is commonly used in Ear, Nose and Throat (ENT), head and neck surgery for coagulation and cutting deeper tissues.

Many studies have highlighted the benefit of diathermy in giving skin incisions but most of the results are not based on ENT, head and neck surgery. ${ }^{2-4}$

This study aim to compare the pain following incision by scalpel and diathermy for skin in ENT surgery.

\section{METHODS}

We conducted a prospective, randomized study in ENT Head and Neck Department, Kathmandu Medical College. Patients undergoing ENT or Head and Neck Surgery at Kathmandu Medical College Teaching Hospital from September 2016 to August 2017 were included in the study who fulfilled the inclusion/exclusion criteria . The study participants were informed about the type of intervention, the procedure of the study, Visual Analog Scale score and written informed consent was taken. The simple randomization technique was used. The study participants were allocated into skin incision by scalpel or skin incision by diathermy group by drawing one of the two cards labeled as "scalpel" or "diathermy" in a sealed envelope. The post operative pain was assessed in post operative $6,12,24$ and 48 hours by using Visual Analog Scale (VAS) score. The total number of on demand analgesics requirement (Ketorolac 30 milligrams per dose intravenous) during 0 to 6 hours, $>6$ to 12 hours, $>12$ to 24 hours and $>24$ to 48 hours were calculated. 
It included the dosage of analgesia administered. Data analysis was done with SPSS software version 11.5 for Windows. The mean was calculated and the statistical test for comparison of mean of VAS score and analgesics requirement in scalpel and electrocautery group was done with Independent $t$ test. $P$ value $<0.05$ was considered statistically significant. The ethical clearance was taken from Institutional Review Committee of Kathmandu Medical College.

The sample size was calculated using the formula , $n=$ $2\left(z_{\alpha}+z_{B}\right)^{2} S^{2} / d^{2}$ where $d=$ difference of mean to be detected $=0.91, z_{a}=1.96$ at $95 \%$ confidence level, $z_{B}=$ 1.28 at $90 \%$ power, $S^{2}=$ pooled variance calculated using $\mathrm{S}_{1}$ and $\mathrm{S}_{2}=1.4849, \mathrm{~S}_{1}=$ scalpel group standard deviation $\mathrm{SD}=1.29, \mathrm{~S}_{2}=$ diathermy group standard deviation $\mathrm{SD}=$ 1.14 and consideration for data loss effect $=10 \%$. The difference of mean and mean standard deviation of pain in post operative 48 hours in scalpel group $\left(\mathrm{S}_{1}\right)$ and diathermy group $\left(\mathrm{S}_{2}\right)$ was taken from a study by $M$ Shamim. ${ }^{2}$ The calculated sample size approximately 62 .

\section{RESULTS}

There were 65 participants out of which 31 were in scalpel group and 30 were in diathermy group. Four participants were excluded from the study. The participants who underwent ear surgery, parotid surgery, neck mass surgery or thyroid surgery at Department of ENT and Head/Neck surgery were included. The participants were randomly allocated into two groups.

The mean age of the participants in scalpel group and diathermy group was $30.4 \pm 13.1$ years and $31.7 \pm 13$. 3years respectively $(P>0.05)$. The male:female ratio in scalpel and diathermy group was 0.8:1 and 2.3:1 respectively $(\mathrm{P}>0.05)$.

The mean VAS score in scalpel group in post operative 6 , 12,24 and 48 hours was $5.4 \pm 1.8,4.8 \pm 1.7,3.5 \pm 1.3$ and $2.5 \pm 1.1$ respectively. The mean VAS score in diathermy group in post operative $6,12,24$ and 48 hours was $3.4 \pm 1.4,3.4 \pm 1.4,2.6 \pm 1.2$ and $1.7 \pm 0.9$ respectively.

The mean analgesic requirement (Ketorolac) in scalpel group in post operative $6,12,24$ and 48 hours was $15.4 \pm 8.7 \mathrm{mg}, \quad 5.8 \pm 2.0 \mathrm{mg}, \quad 6.7 \pm 2.7 \mathrm{mg}$ and $4.8 \pm 1.2 \mathrm{mg}$ respectively. The mean analgesic requirement (Ketorolac) in diathermy group in post operative 6, 12 , 24 and 48 hours was $14.0 \pm 7.1 \mathrm{mg}, 6.0 \pm 4.5 \mathrm{mg}, 1.0 \pm 0.4 \mathrm{mg}$ and $1.0 \pm 0.4 \mathrm{mg}$ respectively.

\section{DISCUSSION}

In 1909, William Bovie was the first person to introduce diathermy in operating room at Harvard University. ${ }^{3}$ Diathermy was later accepted by the surgical world for bloodless cutting and removal of diseased tissues. ${ }^{4}$ Scalpel has been widely used for skin incision. Diathermy is also used these days for making skin incision.

In our study, the mean VAS score decreased in subsequent post operative hours in both scalpel and diathermy group. Similarly, the mean analgesic consumption also decreased in subsequent post operative hours in both the groups.

The mean VAS score was significantly more in scalpel group as compared to diathermy group in post operative 12,24 and 48 hours $(p<0.05)$. The mean VAS score in scalpel group was more than the mean VAS score in diathermy group in post operative hour 6 but it was not statistically significant $(5.4 \pm 1.8$ vs $3.4 \pm 1.4, p>0.05)$.

The mean ketorolac requirement was more in scalpel group as compared to diathermy group in post operative 6 and 48 hours but it was not statistically significant ( $p>0.05$ ), whereas, the mean ketorolac requirement was significantly more in scalpel group than in diathermy group in post operative 24 hours $(6.7 \pm 2.7 \mathrm{vs} 1.0 \pm 0.4$, $\mathrm{p}<0.05)$. Chyros et $\mathrm{al}^{2}$ found that the parenteral analgesic requirement was less in diathermy group as compared to scalpel group in early post operative period which is similar to our findings. However, the mean ketorolac requirement was more in diathermy group as compared to scalpel group in post operative 12 hours which was not statistically significant.

Our study highlights the advantage of skin incision with diathermy in terms of less post operative pain. A similar study have highlighted the role of diathermy in skin incision in terms of incision time, blood loss, wound complications and scar cosmesis. ${ }^{8}$ A study with electrosuergical skin incisions in midline laparotomy have concluded that electrosurgical incisions have significant advantages over scalpel on the basis of early post operative pain and analgesics requirement. ${ }^{5}$ A systematic review and meta-analyis ${ }^{6}$ concluded that electrocautery significantly reduced post operative wound pain. The another meta-analyis ${ }^{7}$ have supported the routine use of diathermy for skin incisions as they have concluded lesser blood loss and lower incidence of early post operative pain with the use of diathermy for skin incisions. A study of bilateral neck dissections concluded that there was no difference in patient satisfaction outcomes when they compared steel scalpel versus electrocautery blade for skin incision. ${ }^{8}$ There was a significant difference in both subjective and objective measurements of pain in patients whose incisions were 
fashioned with an electrosurgical technique compared with those whose were made using a scalpel. ${ }^{9}$ A study comparing the amount of post operative analgesia noted that during the first postoperative day the electrocautery group used half the amount of parenteral analgesia. ${ }^{10} \mathrm{~A}$ study that compared the wound assessed the outcome of patients following use of scalpel or surgical diathermy in elective skin incision of head and neck cancer and concluded that there is no change in wound complication rate and scar formation even after application of heat during use of surgical diathermy. ${ }^{11}$ A similar study compared the diathermy and scalpel in tension free inguinal hernioplasty and concluded that the use of diathermy for skin incision reduces the analgesics requirement in the post operative period. ${ }^{7}$ The findings in this study is similar to other study which have shown that early post operative pain is less in patients with skin incision given by diathermy as compared to skin incisions by scalpel.

The use of monopolar diathermy generates high temperature at the tip and results in marked lateral thermal spread. ${ }^{12}$ The mechanism of lower mean VAS score in diathermy group can be explained by the vaporization effect caused by application of current while using diathermy to incise the skin which leads to immediate tissue and nerve necrosis, consequently, there is total destruction to cutaneous nerves in the area of incision.

The study compares pain in early post operative period up to 48 hours in scalpel and diathermy group in ENT surgery. The study does not compare rate of wound infection or cosmesis in the two groups which are other morbidity parameters requiring a study with a longer follow up period.

\section{CONCLUSIONS}

The early post operative pain is less in ENT-Head and neck surgery patients with skin incision by diathermy as compared to the patients with skin incision by scalpel.

\section{REFERENCES}

1. Sheldon VP, Alastair C, Roy CG. The history of electrosurgery. Dermatol Surg. 2000;26(10):904-8.[PubMed]

2. Shamim M. Diathermy vs. Scalpel Skin Incisions in General Surgery: Double-Blind, Randomized, Clinical Trial. World J Surg 2009; 33:1594-99

3. Goldwyn RM. Bovie: the man and the machine. Ann Plast Surg. 1979;2:135-53
4. Sheldon VP, Alastair C, Roy CG. The history of electrosurgery. Dermatol Surg. 2000;26:904-8

5. S R Kearns, E M Connolly, S McNally, D A McNamara, J Deasy.Randomized clinical trial of diathermy versus scalpel in elective midline laparatomy.Br J Sur.2001;88: 41-4. [PubMed]

6. L N F Aird, CJ Brown. Systematic review and meta-analysis of electrocautery versus scalpel for surgical skin incisions. Am J Sur. 2012;302:216-21.[PubMed]

7. N Zaheer, A Ahmed. Meta-analysis of the Effectiveness of Surgical Scalpel or Diathermy in Making Abdominal Skin Incisions. Ann Surg. 2011;253:8-13.[PubMed]

8. Chau JK, Dzigielewski P, Mlynarek A, Cote DW, Allen H, Harris JR, Seikaly HR. Steel scalpel versus electrocautery blade: comparison of cosmetic and patient satisfaction outcomes of different incision methods.J Otolaryngol Head Neck Surg. 2009 ;38(4):427-33.[PubMed]

9. Hussain SA, Hussain S. Incisions with knife or diathermy and postoperative pain. Br J Surg. 1988; 75: 1179-80. [PubMed]

10. Chrysos E, Athanasakis E, Antonakakis S. A prospective study comparing diathermy and scalpel incisions in tension-free inguinal hernioplasty. Am Surg. 2005;71:326 -9.[PubMed]

11. V Kumar, M Tewari, HS Shukla. A comparative study of scalpel and surgical diathermy incision in elective operations of head and neck cancer. Ind J Cancer.2011; 48 (2): 216-19.[PubMed]

12. P A Sutton, S Awad, A C Perkins, D N Lobo. Comparision of lateral thermal spread using monopolar and bipolar diathermy, the Harmonic Scalpel and the Ligasure.Br J Sur. 2010;97: 428-33 